\title{
Monitoring EBV infection, MSI, PDL-1 expression, Her-2/neu amplification as a biomarker for PD-1 inhibition in gastric cancer
}

\author{
Stanek L ${ }^{1}$, Gurlich R ${ }^{1}$, Musil Z ${ }^{2}$, Havluj L ${ }^{1}$, Whitley A ${ }^{1}$ \\ Third Faculty of Medicine, Charles University in Prague, Department of Surgery, Prague, Czech Republic. \\ stanek.libor@seznam.cz
}

\begin{abstract}
BACKGROUND: The anti-PD-1 monoclonal antibody pembrolizumab has been shown to be associated with a good response in patients with metastatic gastric cancer. Excellent therapeutic results of pembrolizumab have been shown in patients with tumours showing a high microsatellite instability (MSI) and Epstein-Barr virus (EBV) positivity.

GOAL: This is a retrospective study of 40 bioptic specimens from the patients, who underwent gastrectomy for gastric carcinoma. The goal of the study was to identify biomarkers (EBV, MLH-1, PDL-1 expression) that are potentially relevant for selecting the patients, who may benefit from PD-1 inhibition therapy.

METHODS: Immunohistochemical (IHC) expression of PDL-1 and MSI, cytogenetic FISH amplification of the HER-2/neu gene and polymerase chain reaction of EBV RNA, including charge quantification, were performed in selected patients with metastatic or advanced gastric cancer.

RESULTS: EBV-encoded RNA was detected in nine patients. None of them exhibited Her-2 overexpression or CMV infection. PD-L1 was detected in twelve patients. Ten patients were MLH1 positive. All nine cases of EBV infection showed a high expression of PD-L1 and MLH-1 (Tab. 1, Ref. 14). Text in PDF www.elis.sk KEY WORDS: molecular diagnostics, gastric cancer, EBV infection, MSI, PD-L-1 expression.
\end{abstract}

\section{Introduction}

Gastric cancer is an exception among cancers as its incidence and mortality have both significantly decreased over the last 25 years. Its incidence has been reduced by one third and its mortality has been reduced by almost one half. Nevertheless, it remains the fifth most common cancer worldwide.

According to its location, gastric cancer can be classified as cardiac and non-cardiac. In the Western European population, there has been an increase in cardiac tumours with a predominance in men. Cardiac cancer predominates in Central Asia and non-cardiac gastric cancer predominates in East and South Asia. In some countries, including Australia, the United States and the United Kingdom, there is a comparable incidence of both types of cancer, perhaps even a slight predominance of cardiac tumours (1).

Gastric cancer develops through a complex multistage histological process. It is the most common neoplasia of the gastric wall. Its development is influenced by a complex of interactions

${ }^{1}$ Third Faculty of Medicine, Charles University in Prague, Department of Surgery, Prague, Czech Republic, and ${ }^{2}$ First Faculty of Medicine, Charles University in Prague, Institute of Biology and Clinical Genetic, Prague, Czech Republic

Address for correspondence: L. Stanek, Dr, PhD, Third Faculty of Medicine, Charles University in Prague, Department of Surgery, Ruska 87, CZ-100 00 Prague 10, Czech Republic.

Acknowledgements: This work was supported by the Charles University research program PROGRES Q 28 (Oncology). of bacteria ( $H$. pylori), environmental factors, genetic predispositions and molecular mechanisms (2). Much of its aetiology and pathogenesis remain unclear, but the basic context is already known. Histologically, according to the WHO classification, gastric cancer is classified as adenocarcinoma, which accounts for up to $95 \%$ of gastric cancer subtypes (papillary, tubular, mucinous, seal ring-shaped cells), adenosquamous or squamous carcinoma and undifferentiated carcinoma. Early gastric carcinoma is defined as being confined to the mucosa or submucosa, but not growing into the external muscularis. Even this type of tumour has a metastatic potential. The small mucosal subtype only shallowly invades the submucosa and has a diameter of less than 4 $\mathrm{cm}$. The superficially spreading subtype is located in the mucosa with occasional shallow penetrations into the submucosa and has a diameter greater than $4 \mathrm{~cm}$. The pen type penetrates into the submucosa and has a diameter less than $4 \mathrm{~cm}$. It can completely destroy the internal muscularis (Pen A) or preserve it and penetrates it in the form of fenestrations (Pen B). Advanced cancer grows into the muscularis externa or deeper. The Lauren classification distinguishes between two types of gastric cancers, diffuse and intestinal (3). The diffuse type is usually associated with an unfavourable prognosis, as it shows a strong tendency to invade the surrounding tissue. Tumour cells of this type lose cohesion and their original function. The intestinal type has well-defined stages of development and the tumour cells form functional gastrointestinal glands. The intestinal type arises because of chronic atrophic gastritis with intestinal metaplasia of the mucosa and typically appears in older age. The diffuse type arises in younger 
patients without gastritis and metaplasia and consists of signet ring cells in a fibrous or mucosal stroma.

On the molecular level, gastric cancer is a very heterogeneous disease. The Cancer Genome Atlas classifies gastric cancer into four biological groups according to molecular parameters. These are EBV-positive, MSI-high (unstable in microsatellites), chromosomally unstable (CIN) and chromosomally stable (CS), which has the worst prognosis. Each group has a distinct predictive prognostic value, essential for the stratification of treatment options in planned clinical trials. Therapeutic monoclonal antibodies seem to have great potential (4). Heterodimerization of HER-2 and HER-3 receptors is one of the targets of monoclonal antibody therapy. This is because dimerization of HER-2 with other members of the HER family is the greatest driver of tumour cell growth and survival. The human epidermal growth factor (HER) receptor family includes four homologous members (5). Activation of these receptors affects essential tumorigenic processes and plays a crucial role in the pathogenesis of gastric cancer. Trastuzumab is a monoclonal antibody that targets the HER-2 receptors and significantly improves survival, when used in first-line therapy. Ramucirumab, an antibody directed against the vascular endothelial growth factor receptor 2 , has been shown to significantly improve progressionfree and overall survival and has been approved for the second-line treatment of gastric cancer. Anti-mesenchymal-epithelial transition (c-MET), mammalian target rapamycin inhibitors and polo-like kinase 1 inhibitors are under investigation as new treatment options for gastric cancer. These new therapies focus on key immune checkpoint interactions between a T-cell co-inhibitory receptor (PD-1) and one of its immunosuppressive ligands, PD-L1.

In approximately $10 \%$ of gastric cancer cases, cancer cells show ubiquitous and monoclonal Epstein-Barr virus (EBV) infection. A significant feature of EBV-associated gastric cancer (EBVaGC) is a high lymphocytic infiltration, high expression of immune checkpoint proteins, including programmed death-ligand 1 (PD-L1), hypermethlylation of DNA and frequent PIK3CA mutations (6). This highlights EBVaGC as a strong candidate for immune checkpoint blockade therapy. Indeed, several recent studies showed that EBV positivity in gastric cancer correlates with a positive response to programmed cell death protein 1 (PD-1)/ PD-L1 blockade therapy. This can be done directly through the activity of viral proteins and RNA or indirectly by inducing somatic changes in the genome and epigenome of the host organism (6). Understanding the mechanisms that control PD-L1 expression in EBVaGC can indicate new predictive biomarkers for immunotherapy, as well as therapeutic targets for a combination therapy. EBV status is also emerging as a potential biomarker for personalized treatment strategies in gastric cancer. Nevertheless, this subgroup is associated with a better prognosis, thus is less frequently found in advanced or metastatic cancers. In particular, EBV-positive tumours frequently display PD-L1 overexpression, and $\mathrm{T}$ lymphocyte activation. $\mathrm{T}$ cell activation and the extent of the immune response depend on the number of other interactions between receptors and ligands that affect the enhancement or attenuation of T cell activation processes. An important inhibitory pathway that induces T cell anergy is PD-1/PD-L1. This pathway is one of the control points of the immune system and its function is to protect the body against autoimmune disease. At the same time, this pathway may be involved in the development of tumour tolerance. Tumour tolerance is accompanied by an imbalance between costimulatory and inhibitory signals (7). This is a very complex process. If the activity of the PD-1/PD-L1 inhibitory pathway is increased, functional damage to effector T cells may occur, causing them to be “depleted". In this state, lymphocytes are incapable of cytotoxic specific response and tumour cell destruction (8).

MMR deficiency is generally characterized by failure to repair DNA replication-associated errors, leading to the accumulation of mutations in the microsatellite regions of the genome. These phenomena are known as microsatellite instability (MSI). According to the frequency, MSI can be divided into three types: high microsatellite instability (MSI-H), low microsatellite instability (MSI-L) and stable microsatellite (MSS). With the recent development of MSI detection technology and immunosuppressants in the treatment of tumours, researchers have found that tumours with MSI-H respond positively to immunotherapy (9). Immunohistochemical detection evaluates the expression level of MLH-1, from which the degree of MMR damage can be deduced. An important aspect is the length of microsatellites in the normal state and in tumour tissue (10). The occurrence of MSI is the evidence of the MMR mechanism malfunction and may thus predispose to the development of cancer ( $\mathrm{Li}$ et al, 2020). MSI can serve as a biomarker, so its detection is very important. Tumours bearing an MSI change are more likely to respond to treatment with specific drugs, including PD-1 immune checkpoint inhibitors.

\section{Patients}

The median age of the patients was 68 years. All the patients were diagnosed with advanced or metastatic gastric cancer. Most tumours were classified as diffuse adenocarcinoma, adenocarcinoma and intestinal carcinoma. Overall, $97 \%$ of the tumours were poorly differentiated, the rest were moderately or good differentiated. Metastases were present in with lymph node metastases being the most common site.

\section{Methods}

Tumour specimens were obtained from endoscopic biopsies or surgical specimen and were fixed with formalin. Tumour sections were subsequently stained with haematoxylin and eosin. Histologic phenotyping was performed according to World Health Organization (WHO) guidelines. Lymphoepithelioma-like carcinomas were detected during inspection and lymphovascular and perineural invasion were noted when present.

\section{Processing of the tissue}

All specimens were immediately fixed in $10 \%$ formalin, routinely processed and embedded in paraffin. Histological evaluation was performed on slides stained with haematoxylin and eosin using an Olympus microscope (Tokyo, Japan). 
Tab. 1. The results of the immunochemical staining and EBV status.

\begin{tabular}{|c|c|c|c|c|c|c|}
\hline Sample & CMV & EBV & HER-2 & PDL-1 & MLH-1 & Note \\
\hline 1 & neg & neg & neg & neg & positive & instability only \\
\hline 2 & neg & positive & neg & positive & positive & \\
\hline 3 & neg & neg & neg & neg & neg & \\
\hline 4 & neg & neg & neg & neg & positive & instability only \\
\hline 5 & neg & neg & neg & neg & neg & \\
\hline 6 & neg & neg & neg & neg & neg & \\
\hline 7 & neg & neg & neg & neg & neg & \\
\hline 8 & neg & neg & neg & neg & positive & instability only \\
\hline 9 & neg & positive & neg & positive & positive & \\
\hline 10 & neg & positive & neg & positive & positive & \\
\hline 11 & neg & neg & neg & neg & neg & \\
\hline 12 & neg & neg & neg & neg & positive & instability only \\
\hline 13 & neg & neg & neg & neg & neg & \\
\hline 14 & neg & neg & neg & neg & neg & \\
\hline 15 & neg & neg & neg & neg & neg & \\
\hline 16 & neg & positive & neg & positive & positive & \\
\hline 17 & neg & neg & neg & neg & neg & \\
\hline 18 & neg & positive & neg & positive & positive & \\
\hline 19 & neg & neg & neg & positive & positive & mild positivity \\
\hline 20 & neg & neg & neg & neg & neg & \\
\hline 21 & neg & neg & neg & neg & neg & \\
\hline 22 & neg & neg & neg & neg & neg & \\
\hline 23 & neg & neg & neg & neg & neg & \\
\hline 24 & neg & neg & neg & neg & neg & \\
\hline 25 & neg & positive & neg & positive & positive & \\
\hline 26 & neg & neg & neg & neg & neg & \\
\hline 27 & neg & neg & neg & neg & neg & \\
\hline 28 & neg & neg & neg & neg & neg & \\
\hline 29 & neg & positive & neg & positive & positive & \\
\hline 30 & neg & positive & neg & positive & positive & \\
\hline 31 & neg & neg & neg & neg & neg & \\
\hline 32 & neg & neg & neg & neg & neg & \\
\hline 33 & neg & neg & neg & neg & neg & \\
\hline 34 & neg & neg & neg & neg & positive & instability only \\
\hline 35 & neg & neg & neg & neg & neg & \\
\hline 36 & neg & neg & neg & neg & neg & \\
\hline 37 & neg & neg & neg & neg & neg & \\
\hline 38 & neg & positive & neg & positive & positive & \\
\hline 39 & neg & neg & neg & neg & neg & \\
\hline 40 & neg & neg & neg & positive & positive & mild positivity \\
\hline
\end{tabular}

IHC analysis of PD-L1 expression alone was performed using mouse anti-PD-L1 antibody (Abcam). MLH-1. IHC analysis of MLH-1 expression was performed using murine anti-MLH-1 (M1) Primary Antibody (Roche) monoclonal antibodies. In both cases, it was a robotic approach on a BenchMark ULTRA device (Ventana Medical Systems) Roche. The evaluation was performed on an Olympus light microscope (Tokyo, Japan). EBV and CMV Amplification analysis was performed by quantitative multiplex real-time polymerase chain reaction (qPCR), EBV Real-Time PCR (96) KitGen and CMV Real-Time PCR (96) KitGen.

\section{Results}

The results of the immunochemical staining and EBV status of the gastric cancer patients are summarized in Table 1. EBVencoded RNA was detected in nine of the patients (22.5\%), none of which exhibited Her-2 overexpression or CMV infection. PD-L1 was detected in eleven patients (27.5 \%). MLH-1 was detected in sixteen patients (40\%).

High PD-L1 expression was detected in all nine cases of EBV infection. In two additional cases without EBV infection, a mild PD-L1 positivity was detected. In five cases, MLH-1 expression was detected independently of EBV infection and PD-L1 expression.

\section{Conclusion}

Surgical resection supplemented with chemotherapy or chemoradiotherapy remains the only potential curative treatment for gastric cancer. Unfortunately, targeted therapy with the monoclonal therapeutic antibody trastuzumab has not brought a major shift in the management of gastric cancer

HER-2. IHC was performed using monoclonal rabbit antibody against the HER2 protein (clone 4B5, prediluted, Ventana anti-HER2/neu) according to the manufacturer's instructions. Automated HER2 staining was conducted by the immunostainer BenchMark Ultra following the manufacturer's instructions, with an ultraView Universal DAB Detection kit and Bluing reagent as the visualization reagent and chromogen. All materials were obtained from Roche Diagnostics GmbH, Mannheim, Germany.

$P D L-1$. PD-L1 staining was performed to assess the interactions between tumour cells and lymphocytes. PD-L1 in both tumour cells and stroma were recorded. yet as it has in breast cancer. HER2 testing for targeted therapy is currently a part of the basic algorithm in the treatment of breast cancer, and potentially may become a part of the biological treatment in gastric cancer (12). Receptors of the large HER family play an important role in gastric cancer. From a molecular point of view, the therapeutic target is the heterodimerization of HER2 and HER3 receptors. This is because dimerization of HER2 with other members of the HER family is the largest driver of tumour cell growth and survival (11). However, targeted therapy against this receptor is conditioned by amplification of the gene for this Her-2 / neu receptor, which, however, is very sporadic in the case 
of metastatic cancer, occurring in only $5-8 \%$ of cases (12), as evidenced by our results, in which the amplification was ruled out in all the patients $(0 / 40)$. However, immunotherapy with the anti-PD-1 monoclonal antibody pembrolizumab appears to be very promising in the case of tumours with a high microsatellite instability and Epstein-Barr virus (EBV) positivity. Large studies showed that the incidence of EBV infection is relatively high and is detected in approximately $10-20 \%$ of gastric cancers $(13,14)$. Our results (9/40 (22.5\%)) indicate a similar incidence of EBV as in these studies. However, it should be taken in consideration that this was a small cohort of patients, and this may only be a coincidence rather than statistical evidence. An important aspect is that overexpression of PD-L1 programmed cell death ligands has been described in EBV-positive gastric tumours (7). Thus, the Epstein-Barr virus could serve as a potential biomarker in the treatment of gastric cancer with anti-PD-L1 therapy. Positive expression of PD-L1 was observed in the study by Xie et al in 7 out of 9 patients (77.7 \%) diagnosed with gastric cancer and positive for EBV infection. Our analysis showed a positive expression of PD-L1 in 11 out of 40 patients diagnosed with gastric cancer. EBV infection was confirmed in all 9 cases of high PD-L1 expression. In two cases, only mild expression of PD-L1 was detected. In large studies, a similar incidence of PD-L1 expression of about $25 \%$ of the total number of patients with gastric cancer and EBV positivity has been reported $(13,14)$. Patients with gastric cancer were also the subject of the study by Liu et al, who showed that out of a total of 300 patients tested, 27 patients ( $9 \%$ ) showed a very high PD-L1 expression, of which 10 patients had a high MSI and 6 patients were positive for EBV infection. Based on EBV infection, these 6 patients had a high MSIs and strong PD-L1 expression, and these patients are expected to benefit most from immunotherapy. Our results also indicate an association between EBV infection, microsatellite instability and PD-L1 expression. Mild expression of PD-L1 and MLH-1 independent of EBV infection was also demonstrated. In our study, we achieved similar results as in the previous study in terms of finding a mild and high expression and expression associated with EBV infection, but the incidence of individual markers cannot be compared, due to the small cohort of the patients.

\section{References}

1. Ang TL, Fock KM. Clinical epidemiology of gastric cancer. Singapore Med J 2014; 55 (12): 621-628.

2. Shyam Singh, Hem Chandra JHA. Status of Epstein-Barr Virus Coinfection with Helicobacter pylori in Gastric Cancer. J Oncol 2017; 2017: 3456264.

3. Chen YC, Fang WL, Wang RF et al. Clinicopathological Variation of Lauren Classification in Gastric Cancer. Pathol Oncol Res 2016; 22 (1): 197-202.

4. Gravalos C, Jimeno A. HER2 in gastric cancer: a new prognostic factor and a novel therapeutic target. Ann Oncol 2008; 19 (9): 1523-1529.

5. Sergina NV, Moasser MM. The HER family and cancer: emerging molecular mechanisms and therapeutic targets. Trends Mol Med 2007; 13 (12): 527-534.

6. Miliotis CN, Slack FJ. Multi-layered control of PD-L1 expression in Epstein-Barr virus-associated gastric cancer. J Cancer Metastasis Treat 2020; 6 (13): 10.20517/2394-4722.2020.12.

7. Zatloukalova P, Pjechova M, Babcanova S et al. The Role of PD-1/ PD-L1 Signaling Pathway in Antitumor Immune Response. Klin Onkol 2016 Fall; 29 (Suppl 4): 72-77.

8. Barber DL, Wherry EJ, Masopust D et al. Restoring function in exhausted CD8 T cells during chronic viral infection. Nature 2006 Feb 9; 439 (7077): 682-7.

9. Li K, Luo H, Huang L, Luo H, Zhu X. Microsatellite instability: a review of what the oncologist should know. Cancer Cell Int 2020; 20: 16.

10. Samowitz WS, Broaddus R, Iacopetta B, Goldblatt J. PCR versus immunohistochemistry for microsatellite instability. J Mol Diagn 2008; 10 (2): 181-182.

11. Stanek L, Rozkos T, Laco J et al. Comparison of immunohistochemistry, four in situ hybridization methods and quantitative polymerase chain reaction for the molecular diagnosis of HER2 status in gastric cancer: a study of 55 cases. Mol Med Rep 2014; 10 (5): 2669-2674.

12. Uprak TK, Attaallah W, Çelikel CA et al. HER-2 incidence in gastric cancer, its association with prognosis and clinicopathological parameters. Ulus Cerrahi Derg 2015; 31 (4): 207-213.

13. Fukayama M. Epstein-Barr virus and gastric carcinoma. Nihon Rinsho 2012; 70 (10): 1715-1719.

14. Naseem M, Barzi A, Brezden-Masley C et al. Outlooks on EpsteinBarr virus associated gastric cancer. Cancer Treat Rev 2018; 66: 15-22.

Received September 7, 2021. Accepted September 21, 2021. 